\title{
Las acciones proactivas en la búsqueda de patología aórtica aneurismática tienen un impacto en la prevalencia
}

\author{
Proactive measurements in the search of aortic aneurysms have an impact in the \\ prevalence
} Carlos A. Hinojosa*, Karla P. Bermúdez-Serrato, Javier E. Anaya-Ayala, Rafael Pérez-Milán,
Ramón García-Alva y Gerardo Martínez-Méndez

Sección de Angiología, Cirugía Vascular y Terapia Endovascular, Instituto Nacional de Ciencias Médicas y Nutrición Salvador Zubirán, Ciudad de México, México

\begin{abstract}
Resumen
Un aneurisma aórtico abdominal se define como una dilatación focal mayor del 50\% del diámetro normal del vaso. La prevalencia en los adultos mayores de 65 años se estima entre el 2 y el 8\%, con un riesgo de rotura elevado cuando su diámetro es mayor de $5.5 \mathrm{~cm}$ en el sexo masculino y de $5 \mathrm{~cm}$ en el femenino. Este riesgo aumenta linealmente con el crecimiento del aneurisma, y de manifestarse la rotura, la mortalidad puede alcanzar hasta el $80 \%$. La patogénesis y otros factores asociados al crecimiento y la progresión de esta enfermedad no se conocen con certeza, por lo que aislar el aneurisma de la circulación mediante cirugía abierta o endovascular es el objetivo terapéutico para el eliminar el riesgo de rotura. En las últimas décadas se han implementado programas de escrutinio ultrasonográfico para la detección. En el presente artículo hacemos una revisión de la literatura sobre la epidemiología, la evolución natural de los aneurismas y la relevancia de estos programas de escrutinio ultrasonográfico.
\end{abstract}

PALABRAS CLAVE: Aneurisma aórtico abdominal. Escrutinio. Ultrasonido.

\begin{abstract}
An abdominal aortic aneurysm is defined as a focal dilation greater than $50 \%$ of the normal diameter of the vessel. The prevalence in individuals older than 65 years is estimated between the 2 and $8 \%$ with a risk of rupture when the diameter $>5.5 \mathrm{~cm}$ in men and $5 \mathrm{~cm}$ in women. The risk increases exponentially with the expansion of this aneurysmal sac, and if the rupture occurs, its mortality can achieve $80 \%$. The pathogenesis and factors associated to the development and progression of this disease remain not fully understood and isolating the aneurysm from the circulation is the main therapeutic goal to eliminate the risk of rupture. Over the last decades, ultrasonographic screening programs have been implemented for its detection. In this article, we review the epidemiology, natural history of aneurysms and the relevance of ultrasonographic screening programs.
\end{abstract}

KEY WORDS: Abdominal aortic aneurysm. Screening. Ultrasound.

\author{
Correspondencia: \\ ${ }^{*}$ Carlos A. Hinojosa \\ Vasco De Quiroga, 15 \\ Col. Sección XVI, Del. Tlalpan \\ C.P. 14080 , Ciudad de México, México \\ E-mail: carlos.a.hinojosa@gmail.com
}

Fecha de recepción: 09-05-2018

Fecha de aceptación: 24-08-2018

DOI: 10.24875/CIRU.18000445
Cir Cir. 2019;87:470-476

Contents available at PubMed www.cirugiaycirujanos.com 


\section{Introducción}

Un aneurisma es la dilatación focal de una arteria, que tiene riesgo de rotura debido al debilitamiento de sus paredes ${ }^{1}$, por comprometer uno o más segmentos de esta. Se considera dilatación aneurismática aórtica cuando alcanza un diámetro igual o mayor de $3 \mathrm{~cm}$ o una dilatación de al menos 1.5 veces el diámetro esperado ${ }^{2}$. Puede tener forma fusiforme o sacular, y su localización es de mayor prevalencia abdominal que torácica ${ }^{3}$. En la figura 1 puede apreciarse, en una fotografía transoperatoria, un aneurisma aórtico fusiforme. A pesar de tenerse esta como la definición más aceptada, otras definiciones toman el diámetro comparativo de la aorta infrarrenal 1.5 veces el diámetro aórtico suprarrenal, un diámetro mayor o igual que $4 \mathrm{~cm}$ o un diámetro infrarrenal por lo menos $0.5 \mathrm{~cm}$ mayor que el suprarrenal, e incluso se encuentra la definición de Wanhainen, que subdivide a las mujeres con un diámetro mayor o igual que $27 \mathrm{~mm}$ como ya considerado aneurismático. En cuanto a las diferencias derivadas de la edad, el sexo y el índice de masa corporal, el estudio ADAM (The Aneurysm Detection and Management) no encontró diferencias significativas, mientras que otros estudios estiman una diferencia de 0.14 a $0.3 \mathrm{~mm}$ menos en las aortas de mujeres. Debido a la falta de consenso, es importante llevar un seguimiento en los pacientes con factores de riesgo con diámetros de 25-30 mm en adelante, con el objetivo de identificar a los pacientes que presenten efectivamente degeneración aneurismática y normar la conducta a seguir ${ }^{4,5}$.

\section{Epidemiología}

La prevalencia en los hombres de entre 65 y 79 años de edad es del 5 al 10\%", la cual es cuatro a cinco veces mayor que en las mujeres, en las que no se conoce con exactitud la prevalencia, pero en algunos estudios se ha reportado un $1.3 \%{ }^{6}$. Los estudios más recientes muestran al tabaquismo como principal factor de riesgo para su desarrollo; así, el programa de escrutinio de la Administración de Veteranos de los EE.UU. lo ofrece a pacientes con antecedente de tabaquismo o con tabaquismo actual ${ }^{6}$. El tabaquismo incrementa el riesgo de desarrollar un aneurisma aórtico abdominal (AAA) hasta tres veces en comparación con un no fumador incluso 10-19 años después de haberlo dejado, a pesar de disminuir hasta un 30\% las posibilidades de desarrollarlo por cada década $\sin$ fumar, mientras que continuar fumando duplica el riesgo de rotura, con una relación causal y lineal entre la duración y la cantidad de tabaquismo ${ }^{7}$. Históricamente se consideraba que los aneurismas eran resultado de la enfermedad aterosclerótica, pero se ha visto que se trata de una representación focal de una enfermedad vascular generalizada. La presencia de AAA se asocia a arteriomegalia generalizada y ectasia de arterias viscerales, con un perfil molecular y celular distintivo. Por dicho motivo, se ha estudiado el papel que pueda tener la genética para predisponer a las personas, sin encontrar todavía resultados esclarecedores ${ }^{8}$.

La aterosclerosis es un hallazgo común en la pared de los aneurismas, mas no se ha encontrado una relación causal con la formación de estos. Los pacientes con enfermedad cardiaca crónica tienen un mayor diámetro aórtico que aquellos sin enfermedad cardiaca, y se ha establecido un $10-20 \%$ de prevalencia de AAA en enfermos cardiacos crónicos, pero se considera un marcador de riesgo independiente. La presencia paralela de estas afecciones se presume secundaria a factores de riesgo comunes, con distintos mecanismos patogénicos para su desarrollo 9 .

\section{Fisiopatología, factores de riesgo y evolución natural}

Los procesos patológicos involucrados en la formación degenerativa de los AAA incluyen el incremento de las vías de proteólisis, la apoptosis de las células de músculo liso vasculares, el estrés oxidativo, la inflamación y la pérdida de la matriz de la pared arterial ${ }^{8,10}$. Se han estudiado igualmente los cambios hemodinámicos y el impacto en la patogénesis; se considera que las fuerzas de cizallamiento forman parte del estrés que contribuye a desencadenar las cascadas involucradas en la degeneración de la pared aórtica, lo que puede explicar que en su mayoría los AAA se presenten en la aorta infrarrenal por encima de la bifurcación, ya que en esta zona se genera un gradiente de desaceleración sanguínea, con un flujo que cambia de enteramente anterógrado a reverso con recirculación secundario a la mayor resistencia periférica en las extremidades, generando así cambios oscilatorios en la fuerza de cizallamiento de esa zona. La remodelación adapta gradualmente el vaso para evitar alteraciones, pero en personas con predisposición y factores de riesgo puede verse afectado este proceso ${ }^{11}$.

Los hallazgos histológicos en las paredes de AAA incluyen, además de un número reducido de células 
musculares lisas, disminución de la elastina ${ }^{8}$, incremento de células inflamatorias ${ }^{10}$, entre ellas macrófagos, linfocitos Th2 restringidos a CD3+, factor de crecimiento tumoral alfa (TFG $\alpha$ ), interferón gamma y ligando CD40 que estimulan la producción de metaloproteinasas (MMP-2 y MMP-9), y proteasas de cistina para la remodelación vascular'12. La lesión vascular inducida por microorganismos (Chlamydophila pneumoniae, Treponema pallidum y citomegalovirus), cirugía o tabaco puede desencadenar la respuesta inmunitaria causante de la apoptosis y la eventual degeneración de la pared vascular, probablemente por reacción cruzada con proteínas de la pared celular e inflamación ${ }^{12,13}$. Otro mecanismo propuesto del papel del tabaquismo en los aneurismas, aparte de la reacción inflamatoria, es la estimulación de la angiogénesis en el tejido aneurismático que promueve la progresión y rotura del aneurisma ${ }^{13}$.

La aorta abdominal es estructuralmente diferente a la porción torácica. Una de las principales diferencias es la cantidad de capas fibromusculares en cada una, las cuales proveen elasticidad y distribuyen el estrés ejercido en sus paredes. La capa media de la aorta torácica cuenta con alrededor de 60 unidades divididas en regiones vasculares y avasculares, mientras que la abdominal es completamente avascular y cuenta con alrededor de 30 unidades. Debido a las diferencias genéticas detrás de los aneurismas torácicos y aórticos, generalmente no se presentan de manera concomitante, con solo un $10-15 \%$ de probabilidades de que un individuo presente enfermedad aneurismática en ambas porciones de la aorta ${ }^{10}$.

La menor cantidad de unidades lamelares de la capa media, las fuerzas de cizallamiento variables a las que se encuentra sometida constantemente, la predisposición genética y los factores de riesgo modificables que predisponen a las cascadas inflamatorias anormales contribuyen multifactorialmente a la formación de los AAA. Sin embargo, resta entender el desencadenante de los eventos que llevan a la formación de un aneurisma, por lo que, a pesar de existir múltiples estudios dirigidos a localizar genes específicos, así como experimentos para evaluar la acción de distintos medicamentos como estatinas, tetraciclinas, macrólidos, antiinflamatorios no esteroideos e inhibidores de la enzima convertidora de angiotensina que pudieran modificar la evolución natural de la enfermedad, no se ha encontrado ninguna opción farmacológica que evite la progresión, por lo que hasta este momento la cirugía es el tratamiento de elección, $n^{5,8,11-14}$.

Los principales factores de riesgo conocidos son la edad mayor de 60 años, el sexo masculino, contar con antecedentes familiares, el tabaquismo, el colesterol total elevado y el colesterol unido a lipoproteínas de alta densidad disminuido, la hipertensión (en mujeres, sobre todo para rotura), la ingesta elevada de alcohol y la obesidad central como factor de riesgo independiente. La diabetes se ha encontrado como factor protector a pesar de ser un factor de riesgo para aterosclerosis, lo cual apoya la teoría de que esta y los aneurismas son enfermedades independientes que pueden coexistir ${ }^{8}$.

El riesgo de rotura es mayor por arriba de $5.5 \mathrm{~cm}$ en los hombres y de $5 \mathrm{~cm}$ en las mujeres, y aumenta de manera lineal con el diámetro hasta alcanzar una mortalidad asociada del $80 \%{ }^{3}$ y una mortalidad general estimada en un $2 \%{ }^{5,10}$. La tasa anual de rotura para aneurismas con diámetros menores de $4 \mathrm{~cm}$ es del $0.3 \%$, para diámetros de $4-4.9 \mathrm{~cm}$ es del $1.5 \%$, y para diámetros de $5-5.9 \mathrm{~cm}$ es del $6.5 \%$, según las tasas determinadas en el UK Small Aneurysm Trial (UKSAT); solo la mitad de los pacientes que presentan rotura alcanzan a llegar al hospital, y en el resto la mortalidad es del $70-97 \%$. Los pacientes con antecedentes familiares de AAA tienen riesgo de crecimiento acelerado y mayor riesgo de rotura incluso con aneurismas de diámetro menor de $5 \mathrm{~cm}^{5,10}$. El aumento de diámetro de más de $1 \mathrm{~cm}$ por año es indicativo de riesgo de rotura y se utiliza como criterio quirúrgico; un crecimiento anual mayor de $2 \mathrm{~mm}$ predispone a complicaciones asociadas al AAA. Algunas enfermedades de base que se asocian a peor pronóstico en estos pacientes son la enfermedad pulmonar obstructiva crónica, la enfermedad renal crónica, la enfermedad coronaria y la falla cardiaca congestiva ${ }^{8}$.

Con el propósito de disminuir la mortalidad se han realizado múltiples estudios que valoran la utilidad del escrutinio ultrasonográfico en pacientes mayores de 65 años, así como diversas guías de práctica clínica de ámbito internacional; sin embargo, su implementación ha sido muy pobre ${ }^{2}$. Se han descrito tres métodos para la medición ecográfica de la aorta: externo-externo, interno-interno y borde principal-borde principal (borde externo anterior-borde interno posterior), la cual se utiliza sistemáticamente en el ultrasonido cardiaco, siendo esta última la de mayor reproducibilidad interoperador ${ }^{14}$ (Fig. 1).

\section{Reseña cronológica de los estudios de escrutinio ultrasonográfico en aneurismas aórticos abdominales}

El primer estudio de casos y controles se realizó en el Reino Unido en 1995 e incluyó 15,775 hombres y 


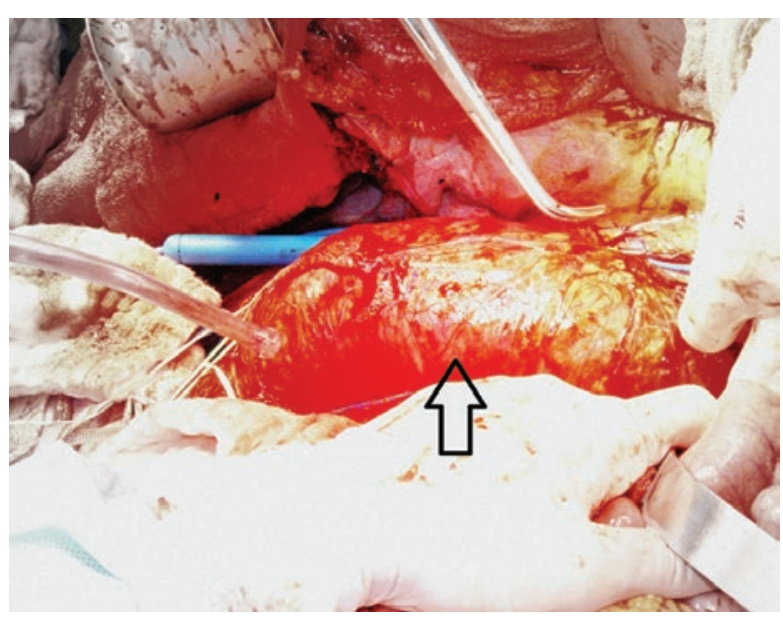

Figura 1. Fotografía transoperatoria en la que se observa un aneurisma aórtico abdominal durante su reparación quirúrgica.

mujeres de 65 a 80 años, que fueron divididos en dos grupos. A los controles se les dio seguimiento sin realizarles escrutinio ultrasonográfico, mientras que a los del otro grupo se les ofreció la prueba de escrutinio. De los 7887 individuos, 5394 aceptaron. Se tomaron como criterios quirúrgicos el diámetro mayor de $6 \mathrm{~cm}$, la expansión anual mayor de $1 \mathrm{~cm}$ y pacientes con sintomatología asociada al aneurisma. Al final se obtuvieron 218 pacientes con AAA, de los cuales 90 cumplían criterios y 31 fueron sometidos a cirugía de aorta. Los resultados mostraron una reducción del $42 \%$ en la mortalidad asociada a la rotura, una disminución del $55 \%$ en la incidencia de rotura con seguimiento a 5 años, y al contar a los controles se observa una reducción en la rotura de hasta el $85 \%$. En las mujeres no se encontraron datos que indiquen una disminución de riesgo relacionado al escrutinio ${ }^{3}$.

El estudio de mayor relevancia a la fecha que se ha realizado es el Multicentre Aneurysm Screening Study (MASS), también en el Reino Unido. Se trata de un ensayo clínico aleatorizado que incluyó 67,800 hombres de entre 65 y 74 años en cuatro centros hospitalarios. Se tomó como punto de corte para indicación quirúrgica $5.5 \mathrm{~cm}$ de diámetro, mientras que a aquellos de $3-5.4 \mathrm{~cm}$ se les dio seguimiento, anual para los de $3-4.4 \mathrm{~cm}$ y trimestral para los de 4.5-5.4 cm. Los resultados fueron similares a los del estudio anterior, con una disminución del riesgo de mortalidad asociada a rotura del $42 \%{ }^{15}$.

En un estudio de cohorte retrospectiva publicado por Ruff, et al. ${ }^{16}$ se analizó el tamizaje intencionado realizado por ultrasonido contra aquel incidental mediante otros métodos de imagen abdominal. A pesar de contar con las recomendaciones de los US Preventive Services Task Force, que indican el escrutinio en pacientes de 65 a 75 años con y sin antecedente de tabaquismo, se observó muy poco apego a ellas.

El ultrasonido abdominal tiene una sensibilidad del $95 \%$ y una especificidad cercana al $100 \%$ para la detección de aneurismas aórticos; sin embargo, el tamizaje se realiza mayormente con otros estudios de imagen, lo cual se asoció en mayor parte a la falta de familiarización con las guías para tamizaje de AAA. Entre otras causas está también el desconocimiento de los pacientes acerca de esta patología, así como su escrutinio y la percepción del médico sobre este ${ }^{16}$.

Se han realizado estudios sobre el tamizaje para AAA en pacientes sometidos a un ecocardiograma, secundando la relación de los factores de riesgo para la presentación de problemas ateroescleróticos en las coronarias y en la en aorta. Dichos estudios encuentran una incidencia del 0.8-6.5\% de prevalencia de AAA, y el escrutinio se lleva a cabo en un tiempo máximo de 7 minutos, lo cual facilita su realización $n^{2,17}$.

Más recientemente, Kevin, et al. realizaron un estudio retrospectivo sobre el tamizaje y el seguimiento de pacientes con aneurismas pequeños, de $3-5.4 \mathrm{~cm}$, y encontraron que únicamente había una continuidad del $65.2 \%$ en un grupo de 568 pacientes, identificando como principales factores de fracaso en el seguimiento la falta de estudios para vigilancia por parte del médico, así como la falta de interés por el paciente y la muerte previa al seguimiento ${ }^{6}$. Es importante notar que dentro de este estudio se encontró un mayor riesgo de muerte secundaria a complicaciones cardiovasculares o cerebrovasculares, las cuales pueden ser prevenidas con un adecuado seguimiento. En esta misma cohorte retrospectiva con base en su programa nacional de escrutinio se encontró como principal factor de riesgo el tabaquismo, con un total del $90 \%$ y una mínima diferencia entre los fumadores activos y aquellos que ya no fumaban, seguido de los sujetos con hipertensión diagnosticada y dislipidemia en tratamiento ${ }^{18}$.

Un subgrupo importante es aquel con diámetro aórtico interno entre 2,5 y $2,9 \mathrm{~cm}$, y con diámetro externo de $30 \mathrm{~mm}$, ya que algunos estudios proponen que estos presentan un $50 \%$ de riesgo de desarrollar un aneurisma aórtico en 5 años ${ }^{14}$.

\section{Escrutinio ultrasonográfico en América Latina}

En varios países latinoamericanos se han realizado estudios de escrutinio para determinar su prevalencia poblacional, todos mediante ecografía abdominal por su alta sensibilidad y especificidad (95\% y $100 \%$, 


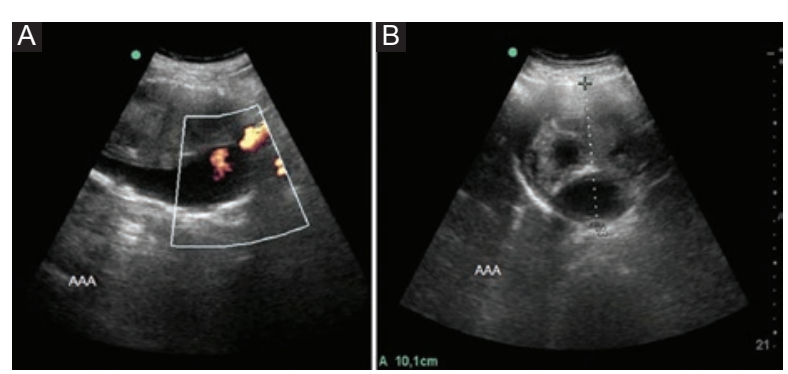

Figura 2. Ecografía abdominal de aorta de un varón de 72 años que acudió para el programa de escrutinio de aorta con aneurisma infrarrenal fusiforme de $10.1 \mathrm{~cm}$ de diámetro máximo anteroposterior, con trombo que ocluye el $80 \%$ del vaso. B con permiso para su reproducción de Permanyer: Predictive value of computed tomographic screening of aortic aneurysms in the Mexican population older than 55 years, Gac Med Mex. 2017;153(Supl. 2):S27-S33.

respectivamente $)^{18}$, y todos con muestras relativamente pequeñas, que sin embargo muestran prevalencias similares a lo reportado en los principales estudios de escrutinio del mundo (MASS, Chichester, Viborg y Western Australia) ${ }^{19}$. En un estudio realizado por Grosso, et al. ${ }^{18}$ en Argentina, en pacientes en quienes se realizó una ecocardiografía transtorácica, se hizo el escrutinio en la aorta abdominal y la prevalencia fue del $10.87 \%$ en varones mayores de 50 años y del $1.47 \%$ en mujeres mayores de 65 años. En Colombia, Poveda y Rojas ${ }^{20}$ reportan un $5.26 \%$ de prevalencia en un total de 113 pacientes evaluados. Batallés, et al. ${ }^{21}$ reportan en Argentina un $5.4 \%$ en varones y un $4.5 \%$ en mujeres entre 184 pacientes de 45 a 87 años. En Brasil, Souto, et al. ${ }^{23}$ realizaron escrutinio en 834 pacientes y encontraron 21 aneurismas (prevalencia del 2.5\%), 15 de ellos en hombres. Finalmente, en Medellín, García, et al. ${ }^{23}$ reportan solo un $0.75 \%$ de aneurismas en un estudio que incluyó 532 individuos.

\section{Contexto de la enfermedad aneurismática en México}

La Guía de Práctica Clínica Mexicana sobre AAA del Instituto Mexicano del Seguro Social refiere que el $48 \%$ de los AAA se descubren clínicamente, el $37 \%$ incidentalmente mediante estudios de imagen solicitados por otra causa y el $15 \%$ durante una intervención quirúrgica abdominal; todos estos datos de acuerdo a las guías de la ACC-AHA (American College of Cardiology - American Heart Association) de $2005^{24}$. Menciona como recomendación la búsqueda intencionada de AAA por parte de radiólogos y clínicos en todo paciente a quien se le realice un ultrasonido, una tomografía computarizada o una resonancia

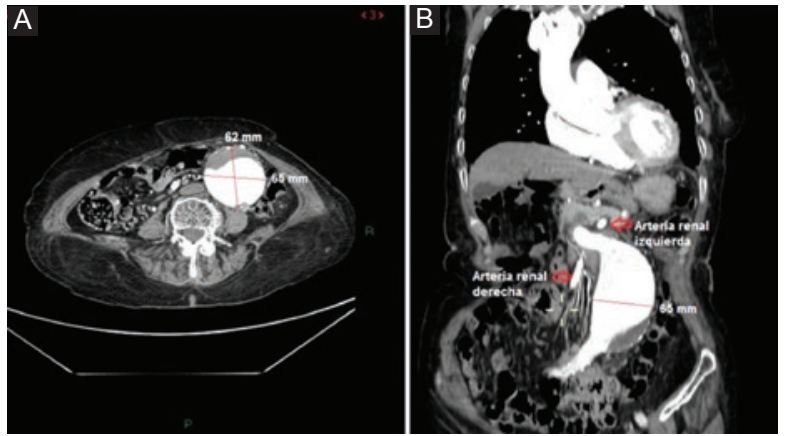

Figura 3. Mujer de 89 años con hallazgo incidental de aneurisma de aorta abdominal infrarrenal fusiforme de $65 \times 62 \mathrm{~mm}$. B con permiso para su reproducción de Permanyer: Predictive value of computed tomographic screening of aortic aneurysms in the Mexican population older than 55 years, Gac Med Mex. 2017;153(Supl. 2):S27-S33.

magnética abdominal. Así mismo, recomienda la realización de ultrasonido para tamiz y seguimiento de AAA en pacientes con factores de riesgo o con sospecha clínica de AAA, incluyendo el diámetro anteroposterior y transversal y la longitud de la lesión, así como la descripción de las ilíacas (Figs. 2 y 3).

Identifica como población de riesgo a los hombres mayores de 55 años cuando haya antecedentes familiares de AAA y a los mayores de 65 aun cuando no los haya. En las mujeres se recomienda para las mayores de 65 en caso de haber antecedentes de AAA. Para los pacientes con diámetro del aneurisma entre 4 y $5.4 \mathrm{~cm}$ hay controversia sobre el seguimiento requerido 24,25 .

En 2015 se llevó a cabo un estudio con 144 pacientes hospitalizados en el Hospital de Especialidades del Instituto Mexicano del Seguro Social, con una media de edad de 72.7 años, y se detectó una prevalencia del $6.9 \%$ en los hombres (95.1\% de la muestra), con lo cual la Institución se reitera en una detección sistemática mediante Doppler dúplex ${ }^{26}$.

A pesar de las recomendaciones de las guías de práctica clínica sobre el escrutinio ultrasonográfico para el AAA, tanto en el país como en el resto del mundo $0^{1,3,14-17,27}$, y de haberse demostrado una disminución en la mortalidad de 4 en 1000 en aquellos pacientes que se someten al estudio, con una reducción de la mortalidad general, contra 1 en 1000 en escrutinio para mama y 0.7 en 1000 para cáncer de colon sin disminución en la mortalidad general ${ }^{27}$, no se han implementado programas en el sector salud ni se realiza habitualmente en los pacientes con factores de riesgo.

En cuanto a costo-efectividad, los estudios de revisión han demostrado una reducción en los costos para el sector salud, aun al disminuir la prevalencia a 


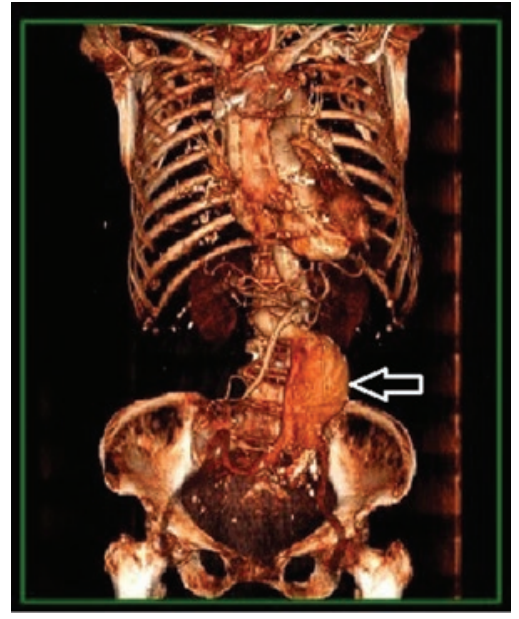

Figura 4. Tomografía computarizada con reconstrucción tridimensional de aneurisma aórtico infrarrenal fusiforme. Con permiso para su reproducción de Permanyer, Predictive value of computed tomographic screening of aortic aneurysms in the Mexican population older than 55 years, Gac Med Mex. 2017;153(Supl. 2):S27-S33.

Tabla 1. Estudios de cohortes más importantes de escrutinio de aneurisma de aorta abdominal y prevalencia encontrada

\begin{tabular}{lcc}
\hline Estudio & Población estudiada & Prevalencia \\
\hline MASS $^{28}$ & 27,204 & $4.9 \%$ \\
Viborg $^{29}$ & 18,749 & $3.3 \%$ \\
Western Australia $^{30}$ & 41,000 & $7.2 \%$ \\
Chichester $^{31}$ & 2212 & $7.7 \%$ \\
Ramírez y PozO $^{21}$ & 113 & $5.26 \%$ \\
Grosso, et al. $^{19}$ & 280 & $4.49 \%$ \\
\hline MASS: Multicentre Aneurysm Screening Study. &
\end{tabular}

la mitad de lo establecido en los estudios iniciales $^{1,27,28}$. En el Instituto Nacional de Ciencias Médicas y Nutrición Salvador Zubirán se implementó desde el mes de octubre de 2015 una campaña de escrutinio ultrasonográfico en pacientes mayores de 65 años con factores de riesgo, en conjunto con las especialidades dentro de la institución que concentran población en riesgo, como los servicios de cardiología, neumología y urología. Hasta la fecha se ha realizado escrutinio en 545 individuos y se ha encontrado una prevalencia del $8.4 \%$, y de estos se diagnosticaron siete aneurismas con indicación quirúrgica. En la figura 2 se muestra la ecografía abdominal de aorta de un paciente varón de 72 años que acudió para el programa de escrutinio de aorta con un aneurisma infrarrenal fusiforme. La figuras 3 y 4 muestran angiotomografías de pacientes a quienes se les había detectado un AAA durante el programa. En la tabla 1 se resumen y comparan los estudios de mayor importancia, tanto internacionales como nacionales.

\section{Conclusiones}

Los estudios realizados en distintas partes del mundo han demostrado prevalencias de entre el 2 y el $10 \%$ en el mismo grupo de riesgo, con lo cual se pone de manifiesto la necesidad de establecer una estrecha comunicación entre las diferentes especialidades médicas para su sospecha, diagnóstico y pronta referencia a los servicios de cirugía vascular. Resulta crucial la implementación de programas de escrutinio en nuestro país que identifiquen a aquellos individuos con esta patología aórtica, y de esta manera reducir la mortalidad asociada.

\section{Conflicto de intereses}

Los autores declaran que no existe conflicto de intereses.

\section{Responsabilidades éticas}

Protección de personas y animales. Los autores declaran que para esta investigación no se han realizado experimentos en seres humanos ni en animales.

Confidencialidad de los datos. Los autores declaran que han seguido los protocolos de su centro de trabajo sobre la publicación de datos de pacientes.

Derecho a la privacidad y consentimiento informado. Los autores han obtenido el consentimiento informado de los pacientes y/o sujetos referidos en el artículo. Este documento obra en poder del autor de correspondencia.

\section{Bibliografía}

1. Cosford PA, Leng GC. Screening for abdominal aortic aneurysm. Cochrane Database Syst Rev. 2007;(2):CD002945.

2. Aboyans V, Bataille V, Bliscaux $P$, Ederhy S, Filliol D, Honton B, et al. Effectiveness of screening for abdominal aortic aneurysm during echocardiography. Am J Cardiol. 2014;114:1100-4.

3. Granel A, Sarandria R. Guía de práctica clínica. Aneurismas de aorta abdominal: ¿está justificado su rastreo sistemático? Evid Actual Pract Ambul. 2005;8:150-3.

4. Wanhainen A. How to define an abdominal aortic aneurysm - influence on epidemiology and clinical practice. Scand J Surg. 2008;97:105-9.

5. Golledge J, Muller J, Daugherty A, Norman P. Abdominal aortic aneurysm: pathogenesis and implications for management. Arterioscler Thromb Vasc Biol. 2006;26:2605-13.

6. Chun KC, Schmidt AS, Bains S, Nguyen AT, Samadzadeh KM, Wilson MD, et al. Surveillance outcomes of small abdominal aortic aneurysms identified from a large screening program. J Vasc Surg. 2016:63:55-61.

7. Norman PE, Curci JA. Understanding the effects of tobacco smoke on the pathogenesis of aortic aneurysm. Arterioscler Thromb Vasc Biol. 2013;33:1473-7.

8. Nordon IM, Hinchliffe RJ, Loftus IM, Thompson MM. Pathophysiology and epidemiology of abdominal aortic aneurysms. Nat Rev Cardiol. 2011:8:92-102.

9. Johnsen SH, Forsdahl SH, Singh K, Jacobsen BK. Atherosclerosis in abdominal aortic aneurysms: a causal event or a process running in parallel? The Tromsø study. Eur J Vasc Endovasc Surg. 2013;45:135-40. 
10. Kuivaniemi H, Ryer EJ, Elmore JR, Tromp G. Understanding the pathogenesis of abdominal aortic aneurysms. Expert Rev Cardiovasc Ther. 2015;13:975-87.

11. Tanweer $\mathrm{O}$, Wilson TA, Metaxa $E$, Riina HA, Meng $H$. A comparative review of the hemodynamics and pathogenesis of cerebral and abdominal aortic aneurysms: lessons to learn from each other. $\mathrm{J}$ Cerebrovasc Endovasc Neurosurg. 2014;16:335-49.

12. Lindholt JS, Shi GP. Chronic inflammation, immune response, and infection in abdominal aortic aneurysms. Eur J Vasc Endovasc Surg. 2006;31:453-63.

13. Li ZZ, Dai QY. Pathogenesis of abdominal aortic aneurysms: role of nicotine and nicotinic acetylcholine receptors. Mediators Inflamm. 2012;2012:103120

14. Björck M, Bown MJ, Choke E, Earnshaw J, Flørenes T, Glover M, et al International update on screening for abdominal aortic aneurysms: Issues and Opportunities. Eur J Vasc Endovasc Surg. 2015;49:113-5.

15. Scott RA, Wilson NM, Ashton HA, Kay DN. Influence of screening on the incidence of ruptured aortic aneurysm: 5-year results of a randomized controlled study. Br J Surg. 1995;82:1066-70.

16. Ruff AL, Teng K, Hu B, Rothberg MB. Screening for abdominal aortic aneurisms in outpatient primary care clinics. Am J Med. 2015;128:283-8.

17. Aboyans V, Kownator S, Lafitte M, Brochet E, Emmerich J, Tribouilloy C, et al. Screening abdominal aorta aneurysm during echocardiography: literature review proposal for a French nationwide study. Arch Cardiovasc Dis. 2010;103:552-8.

18. Grosso OA, Volberg VI, Avalos V, Berensztein CS, Lerman J, Piñeiro DJ. Detección de aneurisma de la aorta abdominal en una población derivada para ecocardiografía transtorácica. Rev Argent Cardiol. 2006;74:217-23.

19. Ramírez-Cabrera J, Pozo-Marcos E Aneurisma de la aorta abdominal: controversias y tendencias en su diagnóstico y manejo. Rev Colomb Cir. 2010;25:323-31.

20. Poveda AG, Rojas DA. Detección temprana de aneurismas de la aorta abdominal mediante escáner dúplex a color. Rev Med. 2007:15:61-7.
21. Batallés SM, Capomasi, M, Longo G, Pezzotto SM, Villavivencio R. Prevalencia de aneurisma de aorta abdominal evaluada con ecografía aórtica y factores de riesgo cardiovascular. Rev Arg Radiol. 2006; 70:285-8.

22. Souto Barros F, Pontes SM, Taylor MA, Roelke LH, Sandri JL, Melo Jacques $\mathrm{Cd}$, et al. Rastreamento do aneurisma da aorta abdominal na população da cidade de Vitória (ES). J Vas Brasileiro. 2005;4:59-65.

23. García G, Calle W, Ramírez W, Díaz C, Timarán R, Uribe JA, et al Prevalencia del aneurisma aórtico abdominal en el área urbana de Medellín, Colombia. latreia. 2008;21:S-16.

24. Hirsch AT, Haskal ZJ, Hertzer NR, Bakal CW, Creager MA, Halperin JL, et al. ACC/AHA 2005 practice guidelines for the management of patients with peripheral arterial disease (lower extremity, renal, mesenteric, and abdominal aortic): executive summary: a collaborative report from the American Association for Vascular Surgery/Society for Vascular Surgery, Society for Cardiovascular Angiography and Interventions, Society for Vascular Medicine and Biology, Society of Interventional Radiology, and the ACC/AHA Task Force on Practice Guidelines (Writing Committee to Develop Guidelines for the Management of Patients With Peripheral Arterial Disease). Circulation. 2006;113:1474-1547.

25. Guía de práctica clínica: aneurismas aórticos abdominales. Catálogo maestro de guías de práctica clínica: IMSS-412-10.

26. Enríquez-Vega ME, Solorio-Rosete HF, Cossio-Zazueta A, Bizueto-Rosas $H$, Cruz-Castillo JE, Iturburu-Enríquez A. Detección oportuna de aneurismas de aorta abdominal en población de riesgo. Rev Med Inst Mex Seguro Soc. 2015;53(Suppl 1):S100-3.

27. Benson RA, Poole R, Murray S, Moxey P, Loftus IM, Ruth A, et al. Screening results from a large United Kingdom Abdominal Aortic Aneurysm Screening Programme Protocols. J Vasc Surg. 2016;63:301-4.

28. Søgaard R, Lindholt JS. Screening for abdominal aortic aneurysm in a modern context and issues for the future. Eur J Vasc Endovasc Surg. 2014:48:668. 